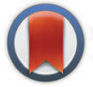

CrossMark \&click for updates

Cite this: Phys. Chem. Chem. Phys., 2016, 18, 26456

Received 9th June 2016, Accepted 1st September 2016

DOI: 10.1039/c6сp04019e

www.rsc.org/pccp

\section{Thermal effect on the morphology and performance of organic photovoltaics $\dagger$}

\begin{abstract}
Eisuke Kawashima, ${ }^{a b}$ Mikiya Fujii ${ }^{\mathrm{ab}}$ and Koichi Yamashita*ab
The morphology of organic photovoltaics (OPVs) is a significant factor in improving performance, and establishing a method for controlling morphology is necessary. In this study, we propose a device-size simulation model, combining reptation and the dynamic Monte Carlo (DMC) algorithm, to investigate the relationship between the manufacturing process, morphology, and OPV performance. The reptation reproduces morphologies under thermal annealing, and DMC showed morphology-dependence of performance: not only short-circuit current density but also open-circuit voltage had optimal interfacial areas due to competition between exciton dissociation and charge collection. Besides, we performed transient absorption spectroscopy of various $\mathrm{BHJ}$ morphologies under realistic conditions, which revealed prompt and delayed dynamics of charge generation-the majority of the charges were from excitons that were generated on interfaces and dissociated within a few picoseconds, and the others from excitons that migrated to interfaces and dissociated on the order of sub-nanoseconds.
\end{abstract}

\section{Introduction}

Organic photovoltaics (OPVs) are promising devices for ubiquitous electricity generation which replace traditional silicon solar cells because of their light weight, flexibility, roll-to-roll printability, ${ }^{1,2}$ and short energy payback time. ${ }^{3}$ However, the power conversion efficiencies (PCEs) of the OPVs are still low (11\% at the highest $\left.{ }^{4}\right)$ and hence optimization schemes are required. The active layers of OPVs are composed of organic donor and acceptor semiconductors: $\pi$-conjugated polymers are frequently used as the donor materials, e.g., poly(3-hexylthiophene) (P3HT), while small molecules such as fullerene derivatives are used as acceptor materials, e.g., $[6,6]$-phenyl- $C_{61}$-butyric acid methyl ester $\left(\mathrm{PC}_{61} \mathrm{BM}\right)$. $\mathrm{P} 3 \mathrm{HT}: \mathrm{PC}_{61} \mathrm{BM}$ is one of the most studied pairs of semiconductors for OPVs, the chemical structures of which are shown in Fig. 1.

The morphology of OPVs - the degree of phase separation, miscibility, and crystallinity of the organic materials-is considered as one of the significant factors for the PCE improvement of OPVS because it strongly affects the efficiencies of exciton dissociation and charge collection described above. In the case of OPVs with a smaller interfacial area and a larger domain, charge carriers are collected efficiently because of transport pathways without bottlenecks, while few excitons dissociate before geminate

\footnotetext{
${ }^{a}$ Department of Chemical System Engineering, Graduate School of Engineering, The University of Tokyo, Tokyo 113-8656, Japan.

E-mail: yamasita@chemsys.t.u-tokyo.ac.jp

${ }^{b}$ CREST, Japan Science and Technology Agency (JST), Tokyo 113-8656, Japan

$\dagger$ Electronic supplementary information (ESI) available. See DOI: 10.1039/c6cp04019e
}

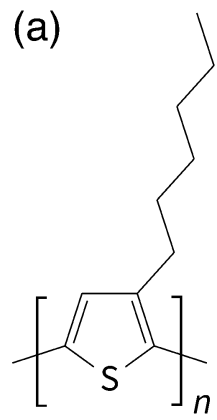

(b)

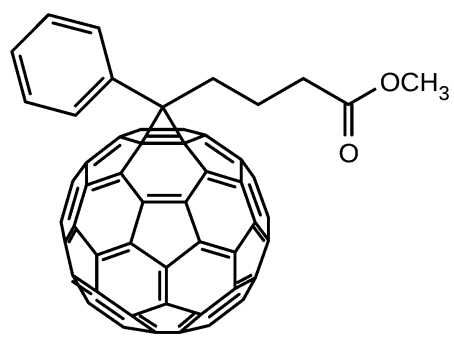

Fig. 1 The chemical structures of typical organic semiconductors: (a) $\mathrm{P} 3 \mathrm{HT}$ and (b) $\mathrm{PC}_{61} \mathrm{BM}$.

recombination. ${ }^{5,6}$ Bulk heterojunction (BHJ) morphology is a breakthrough in OPV development because of its large interfacial area and bicontinuous charge transport pathways. ${ }^{7}$ Morphologies are determined not only by the chemical properties of materials-interaction or solubility-but also by fabrication processes. There have been various experimental studies that investigate how to control morphology to improve the PCE: thermal or solvent annealing, ${ }^{8-11}$ and control of the drying conditions. $^{12,13}$ For instance, it is known that the annealing temperature has an optimum value for efficiency. ${ }^{8,9}$ Transient absorption spectroscopy was also performed to track the efficiency and dynamics of excitons and charge carriers, and influences of morphologies were examined. ${ }^{14}$

Meanwhile, various theoretical studies on OPVs have been presented to obtain a detailed understanding of the mechanism of photocurrent generation. ${ }^{15}$ Quantum chemical calculations 
estimate properties such as energy levels and distributions of molecular orbitals, absorbance, ${ }^{16-19}$ and rate of an elementary process. ${ }^{20}$ However, such molecular-level investigations alone are not sufficient to provide guiding principles for OPV optimization because photocurrent generation is a complicated macroscopic process. Therefore, several theoretical approaches have been presented to investigate morphologies and efficiencies, which are briefly reviewed in the remainder of this section.

To analyze morphologies, molecular dynamics (MD) calculations are performed, which reveal the molecular packing ${ }^{21}$ and molecular orientation of polymers (edge-on and face-on configurations). ${ }^{22}$ Although MD simulations can reveal all morphological properties for OPVs in principle, the computational cost of a device-size system is extremely high even if coarse-grained MD techniques are applied. Another approach is simulations using the Cahn-Hilliard equation, which has been established in soft matter physics. This method requires low computational cost and is applicable to device-size simulation. ${ }^{23-28}$ However, it cannot determine the orientation of polymers because the Cahn-Hilliard equation is a differential equation of concentration of chemical components. Based on the above, we adopt a coarse-grained model called reptation, which has been established to describe polymer melts and solutions. ${ }^{29,30}$ Frost et al. applied reptation to OPVs and revealed that the fraction of polymers would influence quantum efficiency through morphologies. ${ }^{31}$ Reptation also involves low computational cost, but additionally can reveal morphological properties like orientation and miscibility of polymers, and thermal influences on them. ${ }^{31}$ Therefore this method is suitable for studying OPVs, especially the influence of thermal annealing on them.

Several approaches to investigate the PCEs of OPVs have been presented. The simplest one is the equivalent-circuit model, which assumes a solar cell as a circuit of current sources, parallel diodes, and series and parallel resistors. ${ }^{32,33}$ Device simulators known as Scharfetter-Gummel models simultaneously solve coupled equations of electric field and carrier dynamics in the OPVs - the Poisson equation, equation of continuity, and drift-diffusion equation. ${ }^{34-36}$ The last ones are the dynamic Monte Carlo (DMC) algorithm with the first reaction method (FRM) and kinetic Monte Carlo (KMC), which stochastically simulate the dynamics of elementary processes according to the rate. There have been studies which examine morphologydependence of performance-current density and efficiencies of elementary processes such as internal quantum efficiency (IQE) - and dynamics of excitons and charge carriers. ${ }^{27,28,37-46}$ We adopt DMC because it can treat morphologies explicitly and track carrier dynamics in the morphologies.

In this work, we present a combined model of reptation and DMC, which enables the investigation of the relationship between thermal annealing, morphologies, and OPV performances. Morphologies were generated under various conditions by use of reptation, in which current density-voltage $(J-V)$ characteristics were simulated and performance parameters such as short-circuit current density $\left(J_{\mathrm{SC}}\right)$, open-circuit voltage $\left(V_{\mathrm{OC}}\right)$, and PCE were investigated. We showed the existence of optimal annealing conditions for the PCE because of a trade-off between exciton dissociation and charge recombination.

\section{Methodology}

In this article, we present a model of OPVs by combining the two simulation techniques. Morphologies and OPV characteristics were simulated by reptation and DMC, respectively. Various parameters used in these techniques were set to model OPVs of indium tin oxide (ITO)/P3HT: PC $_{61} \mathrm{BM} /$ aluminum, and the simulations were performed at a device scale with a system size of $150^{3} \mathrm{~nm}^{3}$ to perform realistic analyses. The simulation details of reptation and DMC are described below.

\subsection{Reptation}

Reptation, which was introduced by de Gennes ${ }^{29}$ and refined by Doi and Edwards, ${ }^{30}$ is a coarsening model of entangled polymer systems, such as melts or concentrated solutions. The polymer is enclosed in a space called a "tube" and can move easily along the tube, but not perpendicularly to it like a slithering snake.

In this work, polymers were modeled as self-avoiding chains of a certain number of segments connected by bonds, and the excluded volume effect was considered. The system was composed of $150^{3}$ sites, each of which was occupied by either a polymer segment or a small molecule, and the lattice constant was set to $1 \mathrm{~nm}$. Periodic boundary conditions were applied for the $x, y$, and $z$ directions.

The reptation was simulated stochastically with the Metropolis Monte Carlo (MMC) algorithm. ${ }^{31}$ The scheme is shown in Fig. 2. In each MMC step, an end of a polymer tries to move to one of the nearest neighboring empty lattices at random, followed by the rest of the polymer. The acceptance probability $p$ is determined by the temperature $(T)$ and energy change $(\Delta E)$ due to the trial reptation. The total energy of the system, $E$, is given by the sum of interactions between the nearest neighboring sites according to the materials occupying them, while $\Delta E$ is calculated by the interaction change of only the two sites, where the polymer enters and exits, because the other segments of the polymer are unchanged.

Let $n_{\mathrm{p}}$ and $n_{\mathrm{m}}$ be the coordination numbers of polymer segments and small molecules for a site, respectively, and $\varepsilon_{\mathrm{pp}}$, $\varepsilon_{\mathrm{pm}}$, and $\varepsilon_{\mathrm{mm}}$ be polymer-polymer, polymer-small molecule, and small molecule-small molecule interactions, respectively. (a)

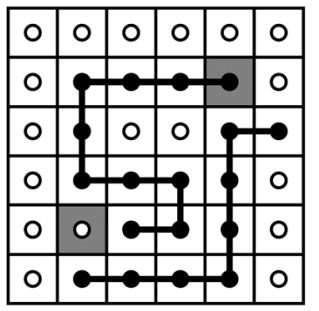

(b)

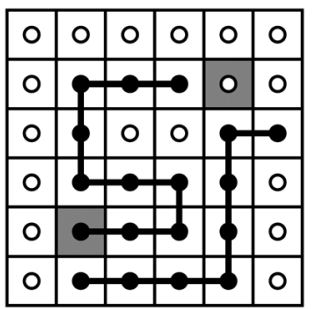

Fig. 2 Schematic representation of reptation in a two-dimensional system: (a) before and (b) after the reptation of a polymer. Each site is occupied by either a polymer segment or a small molecule, which are represented by closed and open circles, respectively, and the polymer segments are connected by bonds represented by lines. Calculation of $\Delta E$ (eqn (3)) requires only the coordination number of polymers and small molecules of the lattices where the polymer enters and exits (the shaded squares). 
Interaction between polymer segments connected by a bond was assumed to be constant and ignored in the calculation of the energy because the length of the polymers was fixed in the simulation. The $\Delta E$ due to reptation is calculated as:

$$
\begin{aligned}
& \Delta E=\Delta E^{\mathrm{en}}-\Delta E^{\mathrm{ex}}, \\
& \Delta E^{\mathrm{en} / \mathrm{ex}}=\left(n_{\mathrm{p}}^{\mathrm{en} / \mathrm{ex}^{\mathrm{ex}}} \varepsilon_{\mathrm{pp}}+n_{\mathrm{m}}^{\mathrm{en} / \mathrm{ex}^{\mathrm{ex}}} \varepsilon_{\mathrm{pm}}\right)-\left(n_{\mathrm{p}}^{\mathrm{en} / \mathrm{ex}} \varepsilon_{\mathrm{pm}}+n_{\mathrm{m}}^{\mathrm{en} / \mathrm{ex}^{\mathrm{ex}}} \varepsilon_{\mathrm{mm}}\right) .
\end{aligned}
$$

Here, the upper indices "en" and "ex" represent those for the site where the polymer enters or exits, respectively. If the coordination numbers of the above site are the same, i.e., $n_{\mathrm{p}}^{\mathrm{en}}+n_{\mathrm{m}}^{\mathrm{en}}=n_{\mathrm{p}}^{\mathrm{ex}}+n_{\mathrm{m}}^{\mathrm{ex}}$, the $\Delta E$ of reptation is simply calculated as

$$
\Delta E=\left(n_{\mathrm{p}}^{\mathrm{en}}-n_{\mathrm{p}}^{\mathrm{ex}}\right) \Delta \varepsilon
$$

with $\Delta \varepsilon \equiv \varepsilon_{\mathrm{pp}}-2 \varepsilon_{\mathrm{pm}}+\varepsilon_{\mathrm{mm}}$; negative or positive $\Delta \varepsilon$ correspond to mutually attractive or mutually repulsive polymers, respectively.

The simulation scheme is as follows: (i) configure polymers randomly in the system (Fig. 5(a)). (ii) Randomly and independently choose a polymer end and direction to reptate that are possible-rechoose them if the pointed site is already occupied by another polymer. (iii) Calculate the energy difference $\Delta E$ of reptation. (iv) Accept the reptation with probability $p$ calculated as

$$
\begin{aligned}
p & =\min \{1, \exp (-\beta \Delta E)\} \\
& =\min \left\{1, \exp \left(-\left(n_{\mathrm{p}}^{\mathrm{en}}-n_{\mathrm{p}}^{\mathrm{ex}}\right) \beta \Delta \varepsilon\right)\right\},
\end{aligned}
$$

where $\beta$ is the inverse temperature $\left(\beta \equiv 1 / k_{\mathrm{B}} T\right.$ with the Boltzmann constant $k_{\mathrm{B}}$ and temperature $T$ ). Reptation is executed if $X<$ $\exp (-\beta \Delta E)$, where $X$ is a uniformly distributed random number in $[0,1)$.

To investigate the dependence of morphologies on annealing temperature and other conditions, reptation was simulated for various $\beta \Delta \varepsilon$, number of polymer segments $n_{\text {seg }}$, and volumetric ratios. The number of polymer segments, $n_{\text {seg }}$, was set to 25,50 , 75 , and 100 , and the volume fraction of polymers to $33 \%$ or $50 \%$; the number of polymers was adjusted to achieve those volume fractions in the simulation volume of $150^{3} \mathrm{~nm}^{3}$. The dimensionless parameter $\beta \Delta \varepsilon$ was set to nonpositive values to simulate mutually attractive or noninteracting polymers or infinite temperature.

\subsection{Dynamic Monte Carlo}

2.2.1 Basic algorithm. DMC simulations were performed to investigate morphology dependencies of photovoltaic characteristics. The system size was unchanged at $150^{3} \mathrm{~nm}^{3}$, while the lattice constant $a$ was increased to $3 \mathrm{~nm},{ }^{37,39,44}$ i.e., the number of sites was decreased to $50^{3}$ to examine three-dimensional devicescale morphologies. We investigated some of the morphologies generated by the reptation $(\mathrm{BHJ})$, and a cube of $3^{3}$ sites was coarsened to one donor or acceptor site depending on the fraction of polymeric donors and molecular acceptors. In addition, some ordered morphologies were investigated for comparisonstriped morphologies with widths of $75 \mathrm{~nm}$ and $15 \mathrm{~nm}$, and checkered morphologies with periods of $75 \mathrm{~nm} \times 75 \mathrm{~nm}, 15 \mathrm{~nm} \times$ $75 \mathrm{~nm}$, and $15 \mathrm{~nm} \times 15 \mathrm{~nm}$ (shown in Fig. 3). Periodic boundary
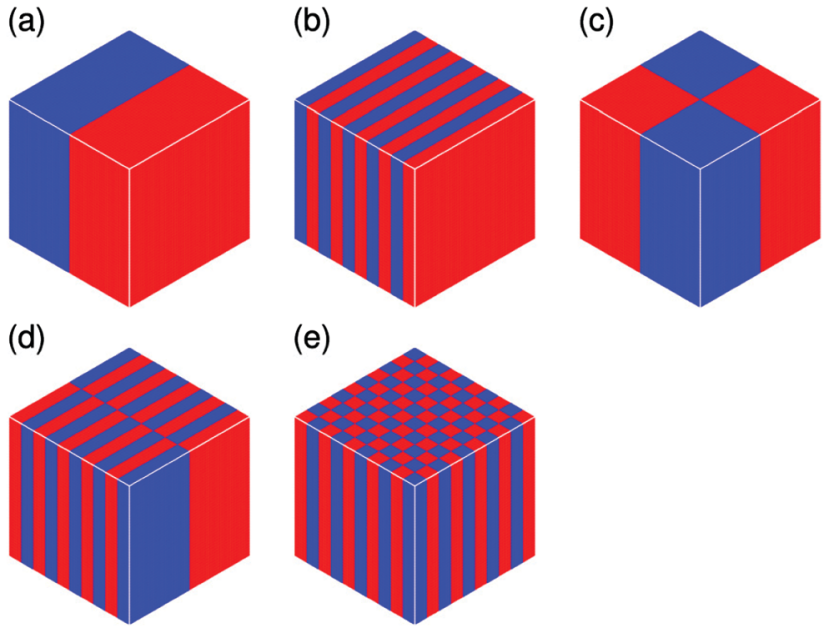

Fig. 3 Ordered morphologies: striped with widths of (a) $75 \mathrm{~nm}$ and (b) $15 \mathrm{~nm}$; checkered with periods of (c) $75 \mathrm{~nm} \times 75 \mathrm{~nm}$, (d) $15 \mathrm{~nm} \times 75 \mathrm{~nm}$, and (e) $15 \mathrm{~nm} \times 15 \mathrm{~nm}$. Blue and red lattices represent donor and acceptor material sites, respectively.

conditions were applied for the $x$ and $y$ directions, and an anode (ITO) and a cathode (aluminum) were set on the top and bottom in the $z$-direction, respectively. Temperature $T$ was fixed at $300 \mathrm{~K}$.

In DMC simulations with the FRM, waiting times of all possible events, calculated from their rate, are stored in a queue; the event holding the minimum waiting time is executed in each DMC step. ${ }^{37,47}$ This work considers three particles, i.e., excitons, holes, and electrons, which are allowed single occupancy of a site. A donor site would be occupied by an exciton or a hole, while an acceptor site by an electron. The simulation scheme is summarized as follows: (i) initialize the system: the first events under photo and dark conditions are exciton generation and charge (hole or electron) injection, respectively. (ii) Insert waiting times of all possible events associated with the particles into the queue; waiting time $\tau$ of each process is calculated as

$$
\tau=-\frac{1}{W} \ln X
$$

where $W$ is the rate of the process and $X$ is a random number uniformly distributed in $(0,1)$. (iii) Pop the event that has the shortest waiting time from the queue and execute it. (iv) Advance the simulation time and reduce waiting times in the queue by the expired time. Insert waiting times of newly possible events into the queue, and remove impossible events from the queue, for example events where single occupancy becomes unsatisfied.

The next subsection explains the details of the stochastic modeling of elementary processes.

2.2.2 Elementary processes. Elementary processes considered in the DMC simulations are (i) generation, (ii) hopping, (iii) dissociation, and (iv) recombination of excitons; (v) injection, (vi) hopping, (vii) collection, (viii) leak, and (ix) recombination of free carriers, i.e., holes and electrons. These processes are 
schematically shown in Fig. 4(a). In particular, events related to carrier transition between the active layer (P3HT:PC $\left.{ }_{61} \mathrm{BM}\right)$ and the electrodes (ITO and aluminum) are summarized in Fig. 4(b). The parameters used in this simulation are listed in Table 1.

2.2.2.1 Excitons. Excitons are bound electron-hole pairs generated by an absorbed photon and have no charge.

(i) In this work AM 1.5 irradiation (power of $100 \mathrm{~W} \mathrm{~cm}^{-2}$ ) was assumed and the exciton generation rate $W_{\mathrm{ex}}^{\text {gen }}$ was set to $8 \times 10^{-10} \mathrm{ps}^{-1} \mathrm{~nm}^{-2}$, which yields a current density of $12.8 \mathrm{~mA} \mathrm{~cm}^{-2}$ when the internal quantum efficiency (IQE) reaches $100 \%$. To save computational resources we insert an event of exciton generation into the queue at a time, without considering where to generate an exciton; at execution of exciton generation an unoccupied donor site is randomly chosen. The probability $p$ is weighted by depth according to the Lambert-Beer law,

$$
p(z) \propto \exp \left(-\alpha\left(z_{\text {top }}-z\right)\right)
$$

where $\alpha$ is the absorption coefficient of the active layer, set to $3 \times 10^{4} \mathrm{~cm}^{-1} \cdot{ }^{49}$

(ii) Once generated, an exciton will hop to a nearby donor site; in this simulation, hopping to all possible sites is considered

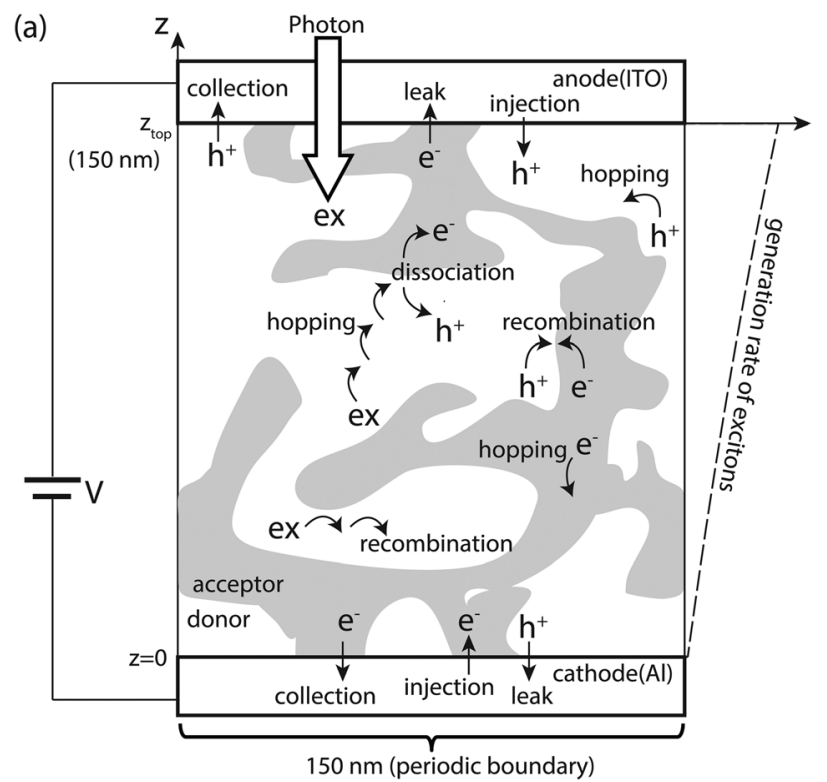

(b)

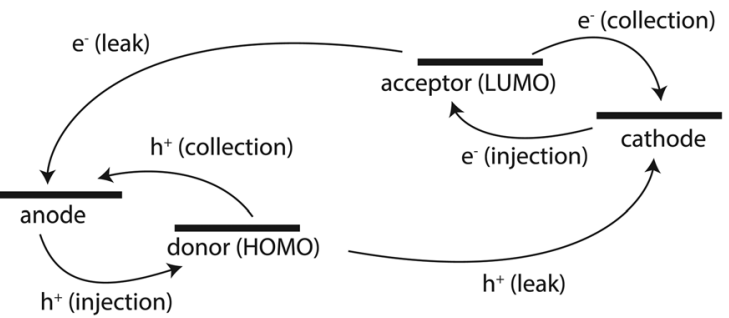

Fig. 4 (a) All elementary processes taken into account in the DMC simulations: (i) generation, (ii) hopping, (iii) dissociation, and (iv) recombination of excitons (represented by ex); (v) injection, (vi) hopping, (vii) collection, (viii) leakage, and (ix) recombination of free carriers, i.e., holes and electrons (represented by $\mathrm{h}^{+}$and $\mathrm{e}^{-}$, respectively). (b) Elementary processes related to carrier transition between the active layer and the electrodes.
Table 1 Parameters used in the DMC simulations

\begin{tabular}{ll} 
Parameter & Value \\
\hline Lattice constant $a$ & $3 \mathrm{~nm}$ \\
Cutoff distance $R_{\text {cut }}$ & $15 \mathrm{~nm}$ \\
Temperature $T$ & $300 \mathrm{~K}$ \\
Relative permittivity $\varepsilon_{\mathrm{r}}$ & 3.5 \\
HOMO of the donor & $-5.0 \mathrm{eV}$ \\
LUMO of the acceptor & $-3.7 \mathrm{eV}$ \\
Work function of the cathode & $4.1 \mathrm{eV}$ \\
Work function of the anode & $4.6 \mathrm{eV}$ \\
Exciton generation rate $W_{\mathrm{ex}}^{\mathrm{gen}}$ & $8 \times 10^{-8} \mathrm{ps}^{-1} \mathrm{~nm}^{-2}$ \\
Exciton lifetime $\tau_{\text {life }}$ & $330 \mathrm{ps}^{48}$ \\
Exciton dissociation rate $W_{\mathrm{ex}}^{\mathrm{dis}}$ & $0.15 \mathrm{ps}^{-120}$ \\
Absorption coefficient $\alpha$ & $3 \times 10^{4} \mathrm{~cm}^{-149}$ \\
Förster radius $R_{0}$ & $3 \mathrm{~nm}^{20}$ \\
Reorganization energy $\lambda$ & $0.11 \mathrm{eV}^{20}$ \\
Gaussian width of DOS $\sigma$ & $0.062 \mathrm{eV}^{39}$ \\
Hole mobility $\mu_{\mathrm{h}}$ & $0.1 \mathrm{~cm}^{2} \mathrm{~V}^{-1} \mathrm{~S}^{-150}$ \\
Electron mobility $\mu_{\mathrm{e}}$ & $0.025 \mathrm{~cm}^{2} \mathrm{~V}^{-1} \mathrm{~s}^{-151}$ \\
Charge recombination rate $W_{\mathrm{ch}}^{\mathrm{rec}}$ & $1.93 \mathrm{~ns}^{-120}$
\end{tabular}

and the rate $W_{\mathrm{ex}}^{\text {hop }}$ is calculated by the Förster energy-transfer theory, $^{52}$

$$
W_{\mathrm{ex}}^{\mathrm{hop}}=\frac{1}{\tau_{\text {life }}}\left(\frac{R_{0}}{R}\right)^{6} \min \{1, \exp (-\beta \Delta E)\},
$$

where $\tau_{\text {life }}$ is the exciton lifetime, $\Delta E$ is the energy change due to the hopping, and $R_{0}$ and $R$ are the Förster radius and hopping distance, respectively. Because an exciton is neutral (always $\Delta E=0$ ), the rate is simply calculated as

$$
W_{\mathrm{ex}}^{\mathrm{hop}}=\frac{1}{\tau_{\text {life }}}\left(\frac{R_{0}}{R}\right)^{6} .
$$

The lifetime, $\tau_{\text {life, }}$, and Förster radius, $R_{0}$, were set to $330 \mathrm{ps},{ }^{48}$ and $3 \mathrm{~nm}$, respectively, so that the diffusion length of excitons was in the order of $10 \mathrm{~nm}$. A cutoff was introduced to reduce the computational cost, i.e., exciton hopping was considered only for sites within the cutoff distance $R_{\text {cut }}=15 \mathrm{~nm}$.

(iii) When an exciton reaches the interface, it is able to dissociate into a hole and an electron. In the simulation all possible donor-acceptor nearest neighboring pairs were considered, and after exciton dissociation a hole was created on the donor site that the dissociated exciton occupied, and an electron on the acceptor site across the interface. The exciton dissociation rate, $W_{\mathrm{ex}}^{\mathrm{dis}}$, was set to $0.15 \mathrm{ps}^{-1}$, which was estimated by quantum chemical calculations. $^{20}$

(iv) Otherwise, an exciton decayed to the ground state and geminate recombination occurs. The exciton recombination rate is the reciprocal of the exciton lifetime: $W_{\mathrm{ex}}^{\mathrm{rec}}=1 / \tau_{\text {life }}$.

2.2.2.2. Holes and electrons. Holes and electrons have charge and hence their transport is affected by the difference of site energy, $\Delta E$, before and after the event. The site energy is a summation of the following three factors:

1. On-site energies of charge carriers (holes/electrons), which are a summation of the orbital energy (HOMO of the acceptor/LUMO of the donor) and Gaussian disorder of density of states (DOS) caused by residual solvents and structural disorder. The HOMO of the acceptor, LUMO of the donor, 
and Gaussian width $\sigma$ were set to $-5.0 \mathrm{eV},-3.7 \mathrm{eV}$, and $0.062 \mathrm{eV},{ }^{39}$ respectively.

2. The Coulomb interaction, $U$, with other charge carriers and image charges, including that of itself. The Coulomb interaction of carrier $i$ is calculated as

$$
U_{i}=\sum_{j \neq i} \frac{1}{4 \pi \varepsilon} \frac{q_{i} q_{j}}{r_{i j}}
$$

where $\varepsilon$ is permittivity, $q_{i}$ is the charge of carrier $i$, and $r_{i j}$ is the distance between carriers $i$ and $j$; the minimum image convention was applied, namely

$$
r_{i j}=\min _{n_{x}, n_{y}} \sqrt{\left(x_{j}-x_{i}+n_{x} L_{x}\right)^{2}+\left(y_{j}-y_{i}+n_{y} L_{y}\right)^{2}+\left(z_{j}-z_{i}\right)^{2}},
$$

where $n_{x, y}$ is the integer in $\{-1,0,+1\}$, and $L_{x, y}$ is the length of the simulation box along $x$ and $y\left(L_{x}=L_{y}=150 \mathrm{~nm}\right)$. The relative permittivity $\varepsilon_{\mathrm{r}}$ was set to 3.5 as a typical value for OPVs.

3 . The electrostatic built-in potential, $V_{\text {built }}$, which is induced by the applied voltage $V$ and the difference in the work functions of the electrodes $(\Delta W=0.5 \mathrm{~V})$ :

$$
V_{\text {built }}=(e V-\Delta W) \frac{z}{z_{\text {top }}}
$$

with elementary charge $e$.

(v) A charge carrier was created in the active layer by either exciton dissociation or injection from the electrode; a hole (an electron) is injected from the anode (cathode) to one of the donor (acceptor) sites at the surface. These injected carriers contribute to the dark current, which is enhanced by the applied voltage, $V$. The charge injection rate $W_{\mathrm{h} / \mathrm{e}}^{\mathrm{inj}}$ is calculated by the Miller-Abraham equation ${ }^{53}$ as

$$
W_{\mathrm{h} / \mathrm{e}}^{\mathrm{inj}}=\frac{6 \mu_{\mathrm{h} / \mathrm{e}} V_{T}}{a^{2}} \min \{1, \exp (-\beta \Delta E)\},
$$

where $\mu_{\mathrm{h} / \mathrm{e}}$ is the hole/electron mobility of pristine donor/ acceptor materials, $V_{T}$ is the thermal voltage $\left(V_{T} \equiv k_{\mathrm{B}} T / e\right)$, and $a$ is the lattice constant. Hole and electron mobilities, $\mu_{\mathrm{h}}$ and $\mu_{\mathrm{e}}$, were set to $0.1 \mathrm{~cm}^{2} \mathrm{~V}^{-1} \mathrm{~s}^{-150}$ and $0.025 \mathrm{~cm}^{2} \mathrm{~V}^{-1} \mathrm{~s}^{-1}, 51$ respectively. The prefactor is derived from the Einstein relationship. ${ }^{37,54}$

In calculations of the energy difference $\Delta E$, the Gaussian disorder of an electrode was not considered. In addition to the above three factors, $\Delta E$ included the charge injection barrier of $+0.4 \mathrm{eV}$, which is derived from the difference between the LUMO of the acceptor $(-3.7 \mathrm{eV})$ and the Fermi level of the cathode $(-4.1 \mathrm{eV})$. Although an injected carrier could be collected from the electrode, it was forced to hop at once before extraction if possible.

(vi) Holes and electrons inside the active layer are allowed to hop to one of the nearest neighboring donor and acceptor sites, respectively. The charge hopping rate $W_{\mathrm{h} / \mathrm{e}}^{\text {hop }}$ is calculated using the Marcus formula as

$$
W_{\mathrm{h} / \mathrm{e}}^{\mathrm{hop}}=\frac{6 \mu_{\mathrm{h} / \mathrm{e}} V_{T}}{a^{2}} \exp \left(\frac{\beta \lambda}{4}\right) \exp \left(-\beta \lambda\left(1+\frac{\Delta E}{\lambda}\right)^{2}\right),
$$

where the reorganization energy $\lambda$, equivalent to twice the polaron-binding energy, was set to $0.11 \mathrm{eV}^{20}$

(vii) When a charge carrier reaches the electrode surface, it is able to be collected and then the current flows. The charge collection rate is also calculated by the Marcus formula (eqn (14)), but the negative of the charge injection barrier is considered for $\Delta E$. Collection of holes from the cathode and electrons from the anode contribute to the photocurrent.

(viii) Leakage of charge carriers, i.e., extraction of holes/ electrons from the anode/cathode, is simulated in the same way as above, but it contributes to the dark current.

(ix) Charge recombinations are considered when a hole and an electron are located on adjacent sites. The rate $W_{\mathrm{ch}}^{\mathrm{rec}}$ was set to $1.93 \mathrm{~ns}^{-1}$. $^{20}$

2.2.3 OPV characterization. DMC simulations were performed for both photocurrent and dark current. No exciton is generated in the latter case while excitons are generated in the former case according to the procedure explained previously. Performance parameters such as $J_{\mathrm{SC}}, V_{\mathrm{OC}}$, IQE, and PCE were calculated for the morphologies. Charge injection (holes/electrons from the anode/cathode) and leakage (holes/electrons extracted to the cathode/anode) contribute to the dark current. Therefore, the net number of collected holes at the anode $n_{\mathrm{h}}^{\text {net coll }}$ (electrons at the cathode $n_{\mathrm{e}}^{\text {net coll }}$ ) is the number of collected holes $n_{\mathrm{h}}^{\text {coll }}$ (electrons $n_{\mathrm{e}}^{\text {coll }}$ ) reduced by those of injected holes $n_{\mathrm{h}}^{\text {inj }}$ (electrons $n_{\mathrm{e}}^{\text {inj }}$ ) and leaked electrons $n_{\mathrm{e}}^{\text {leak }}$ (holes $n_{\mathrm{h}}^{\text {leak }}$ ):

$$
n_{\mathrm{h} / \mathrm{e}}^{\text {net coll }}=n_{\mathrm{h} / \mathrm{e}}^{\text {coll }}-n_{\mathrm{h} / \mathrm{e}}^{\text {inj }}-n_{\mathrm{e} / \mathrm{h}}^{\text {leak }}
$$

and the net number of collected charges is calculated as

$$
n_{\mathrm{ch}}^{\text {net coll }}=\frac{n_{\mathrm{h}}^{\text {net coll }}+n_{\mathrm{e}}^{\text {net coll }}}{2} .
$$

The efficiencies of exciton dissociation $\eta_{\mathrm{ex}}^{\text {diss }}$ and charge collection $\eta_{\mathrm{ch}}^{\text {coll }}$ were calculated as

$$
\eta_{\mathrm{ex}}^{\mathrm{diss}}=\frac{n_{\mathrm{ex}}^{\mathrm{dis}}}{n_{\mathrm{ex}}^{\mathrm{gen}}} \quad \text { and } \quad \eta_{\mathrm{ch}}^{\mathrm{coll}}=\frac{n_{\mathrm{ch}}^{\mathrm{net} \text { coll }}}{n_{\mathrm{ex}}^{\mathrm{dis}}}
$$

respectively, where $n_{\mathrm{ex}}^{\mathrm{gen}}$ and $n_{\mathrm{ex}}^{\mathrm{dis}}$ are the number of generated and dissociated excitons, respectively. The IQE is the product of $\eta_{\mathrm{ex}}^{\mathrm{diss}}$ and $\eta_{\mathrm{ch}}^{\text {coll }}$.

The net current density $J$ is calculated as the number of net collected charges divided by the incident area $\left(150^{2} \mathrm{~nm}^{2}\right)$ and simulation time. The $J-V$ curves were obtained by repeating the calculation of current density under each applied voltage. Open-circuit voltage $V_{\mathrm{OC}}$, PCE, and fill factor (FF) were obtained by linear interpolation of the $J-V$ curve.

Notably, this study also analyzed transient absorption spectroscopy. In the simulation, a certain number of excitons were created at the start under the conditions that no charge injection, leak, nor collection and no applied voltage were imposed. The initial number of excitons was set to 100 and 1000: the latter corresponds to a density of $3 \times 10^{17} \mathrm{~cm}^{-3}$, which is consistent with the experimental value; ${ }^{14}$ the former was for comparison, and note that we can simulate dilute conditions that experimental observation is difficult. There have been studies that discuss the dynamics of carriers-the effect of mobility on 
charge separation, ${ }^{45}$ and the kinetic model of charge separation and recombination. ${ }^{46}$ In this work we examined the dependence of transient absorption spectroscopy on BHJ morphology in consideration of annealing temperature and analyzed spacetime dynamics.

Five simulation runs were performed independently for each condition $-0.5 \mathrm{~ms}$ and $10 \mathrm{~ns}$ for a simulation of current density and transient absorption spectroscopy, respectively-and the results were averaged.

\section{Results and discussion}

\subsection{Reptation}

The generated morphologies with a 1:1 volume ratio and 75 polymer segments are shown in Fig. 5, whose phase separation is consistent with an experimental report ${ }^{55}$ and theoretical studies using the Cahn-Hilliard equation. ${ }^{23-28}$ See Fig. S1 and $\mathrm{S} 2$ in the $\mathrm{ESI} \dagger$ for the other morphologies-those of different volume ratios and number of segments. Generally, the domain size grew as $\beta \Delta \varepsilon$ increased. The temperature increased under the negative constant $\Delta \varepsilon$, while the polymers dispersed when the temperature was too high. These morphologies are characterized numerically: interfacial area per volume and mean distribution of domain size, shown in Fig. 6(a) and (b). Here, the domain size is defined as the length of continuous donor/ acceptor domains along $x, y$, or $z$. Both characteristics depended strongly on $\beta \Delta \varepsilon$ but only slightly on the polymer length. The interfacial area and domain size indicate the minimum and maximum values, respectively, around $\beta \Delta \varepsilon=-1$ because of the (a)

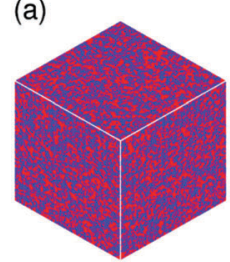

(d)

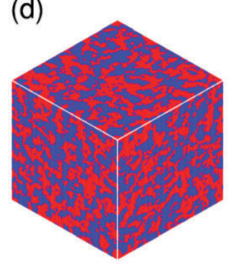

(g)

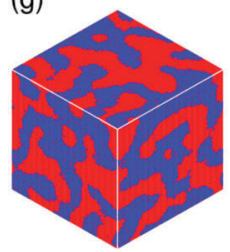

(b)

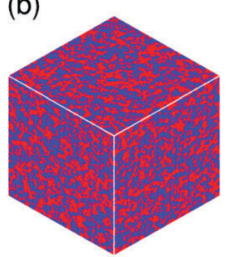

(e)

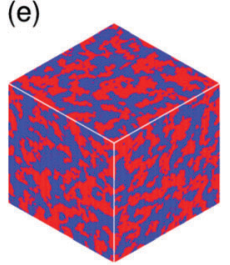

(h)

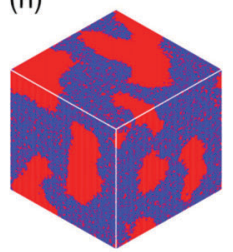

(c)

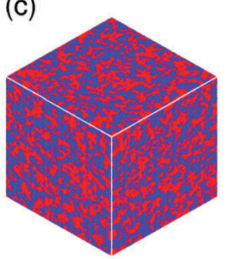

(f)

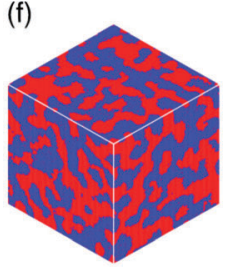

(i)

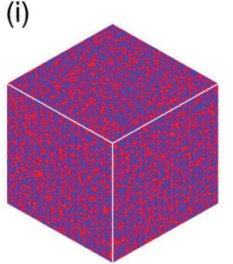

Fig. 5 (a) An initial morphology. Morphologies with $\beta \Delta \varepsilon$ of (b) -4.0 , (c) -3.0 , (d) -2.0 , (e) -1.5 , (f) -1.0 , (g) -0.75 , (h) -0.5 , and (i) 0 . The volume ratio is $1: 1$, and the number of polymer segments, $n_{\text {seg, }}$ is 75 . Blue and red sites represent polymers and small molecules, respectively. Considering the constant $\Delta \varepsilon, \beta \Delta \varepsilon=0$ corresponds to the high temperature limit.

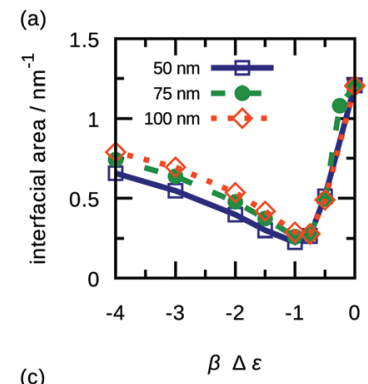

(c)
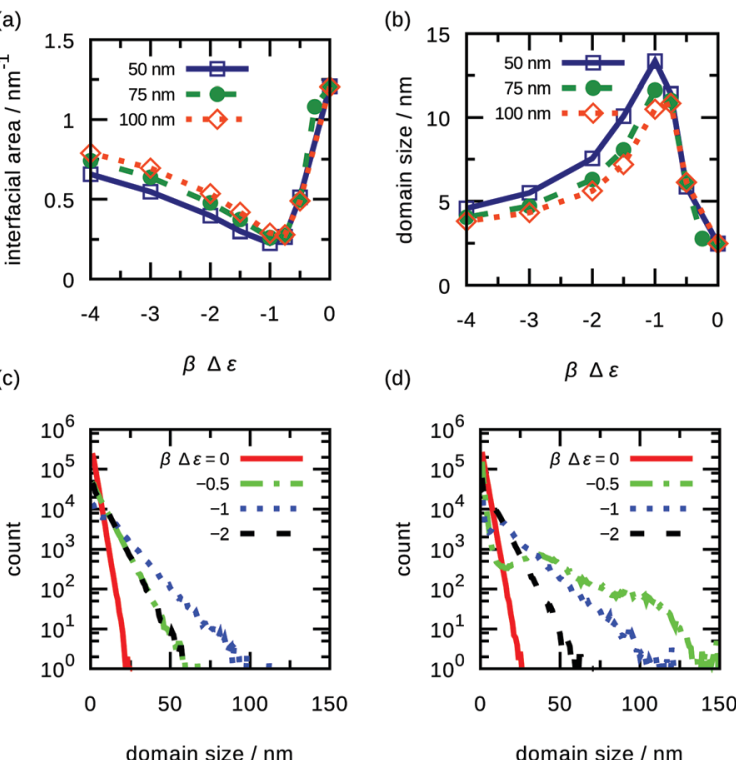

(d)

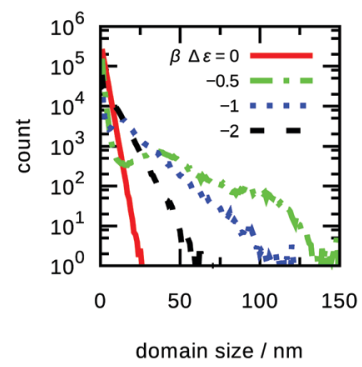

Fig. $6 \beta \Delta$ dependence of (a) interfacial area and (b) mean domain size of donor; domain-size distribution of (c) polymer and (d) small-molecule regions for morphologies of $n_{\text {seg }}=75$. The volume ratio is $1: 1$.

degree of diffusion. At low temperature $(\beta \Delta \varepsilon=-4.0$, shown in Fig. 5(i)), the polymers are hindered in diffusing and trapped in a local minimum because of the attractive interaction. As the temperature rises, the polymer can diffuse and relax, therefore donor domains are formed; however, free diffusion breaks the domains when the temperature is too high. The polymer length also affects diffusion and therefore shows a similar dependence. See Section S1.2 in the ESI $\dagger$ for the results of a polymer/small molecule volumetric ratio of $1: 2$. The interfacial area and domain size were smaller and their dependence on $\beta \Delta \varepsilon$ became milder than those of a $1: 1$ ratio, due to the lower polymer fraction and weaker entanglement effects.

The domain-size distributions of the polymers and small molecules are shown in Fig. 6(c) and (d), respectively. Essentially, the distributions of the domain size for both polymers and small molecules exhibit exponential decay and there is no significant difference between polymers and small molecules. However, the morphology of $\beta \Delta \varepsilon=-0.5$ has significantly different characters: the domains of small molecules in that morphology are larger than those of polymers, and exhibit exceptionally oscillating properties. This was because polymer aggregation precluded acceptors and formed large acceptor domains but polymer domains were broken due to diffusion.

The results in this subsection propose that the morphologies of OPVs strongly depend on the temperature by using the physical modeling of blends of polymers and small molecules.

\subsection{Dynamic Monte Carlo}

For $\mathrm{BHJ}$ morphologies, we chose those with a $1: 1$ volumetric ratio and 75-segment polymers, $n_{\text {seg }}=75$, to investigate wide ranges of interfacial area and domain size (see Fig. 5 and 6). It is expected that other morphology groups-different $n_{\text {seg }}$ and/or volumetric fraction-show a similar but milder temperature dependence. 
For example, morphologies with a 1:2 volume ratio have smaller donor domains, which probably enhances the exciton dissociation efficiency, but lower donor fraction yields fewer excitons and thus their effect on current density is likely canceled.

3.2.1 Performance parameters. Current density-voltage $(J-V)$ characteristics are shown in Fig. 7. Since the sample standard deviations were small (ranging from $0.03 \mathrm{~mA} \mathrm{~cm} \mathrm{~cm}^{-2}$ to $0.3 \mathrm{~mA} \mathrm{~cm}{ }^{-2}$, see Section S2.1 in the ESI $\dagger$ ), the error bars were omitted for clarity. Both photo- and dark currents depended on the morphologies. Interfacial areas of the coarsened morphologies used in these DMC simulations are listed in Table 2, and interfacial area dependencies of performance parameters-short-circuit current density $J_{\mathrm{SC}}$, open-circuit voltage $V_{\mathrm{OC}}, \mathrm{PCE}$, and FF-are shown in Fig. 8. The $J_{\mathrm{SC}}, V_{\mathrm{OC}}$, and PCE exhibit maximum values related to the interfacial area, while the FF decreases monotonically as the interfacial area increases. Different $\mathrm{BHJ}$ morphologies show different dark currents (Fig. 7(c)), while the ordered ones show similar tendencies (Fig. 7(d)). This is because all of ordered morphologies have suitable charge transport paths and charge recombination merely occurs. Photocurrent, shown in Fig. 7(a) and (b), is determined by exciton dissociation and charge collection, whose details are described in the next paragraph. This study shows the morphology dependence of $V_{\text {OC }}$, which arises from the morphology dependence of the photo- and dark currents.

Efficiencies of exciton dissociation and charge collection, and the IQE under short-circuit conditions are shown in Fig. 8(e), (f), and (g), respectively. Their sample standard deviations were smaller than 0.02. In the morphologies with large interfacial areas, the exciton dissociation is enhanced, but the charge collection is discouraged by a longer transport path. Therefore, the IQE
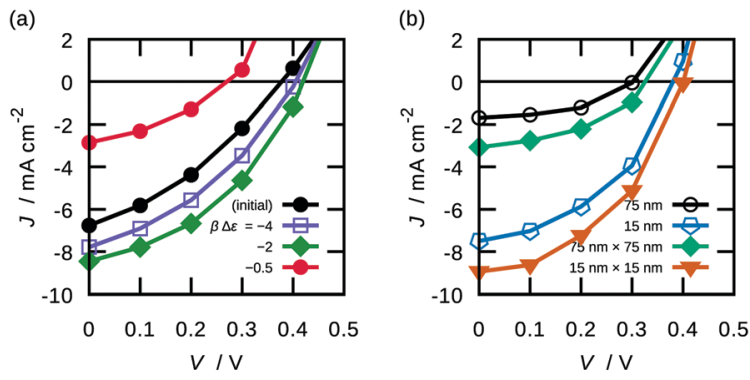

(c)
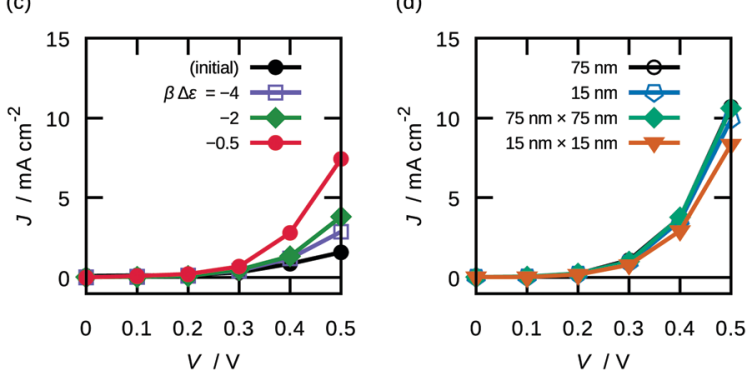

Fig. 7 Current densities for different morphologies and conditions: photocurrent densities of (a) the $\mathrm{BHJ}$ morphologies generated by the reptation and (b) the ordered morphologies; dark current densities of (c) the $\mathrm{BHJ}$ morphologies generated by the reptation and (d) the ordered morphologies.
Table 2 Interfacial area per volume of the morphologies

\begin{tabular}{lll}
\hline Morphology & & Interfacial area $/ \mathrm{nm}^{-1}$ \\
\hline BHJ & Initial & 0.418 \\
& $\beta \Delta \varepsilon=-4.0$ & 0.357 \\
& -3.0 & 0.325 \\
& -2.0 & 0.267 \\
& -1.0 & 0.153 \\
& -0.5 & 0.077 \\
Stripe & 0.0 & 0.482 \\
& $75 \mathrm{~nm}$ & 0.013 \\
Checker & $15 \mathrm{~nm}$ & 0.067 \\
& $75 \mathrm{~nm} \times 75 \mathrm{~nm}$ & 0.027 \\
& $15 \mathrm{~nm} \times 75 \mathrm{~nm}$ & 0.080 \\
& $15 \mathrm{~nm} \times 15 \mathrm{~nm}$ & 0.133
\end{tabular}
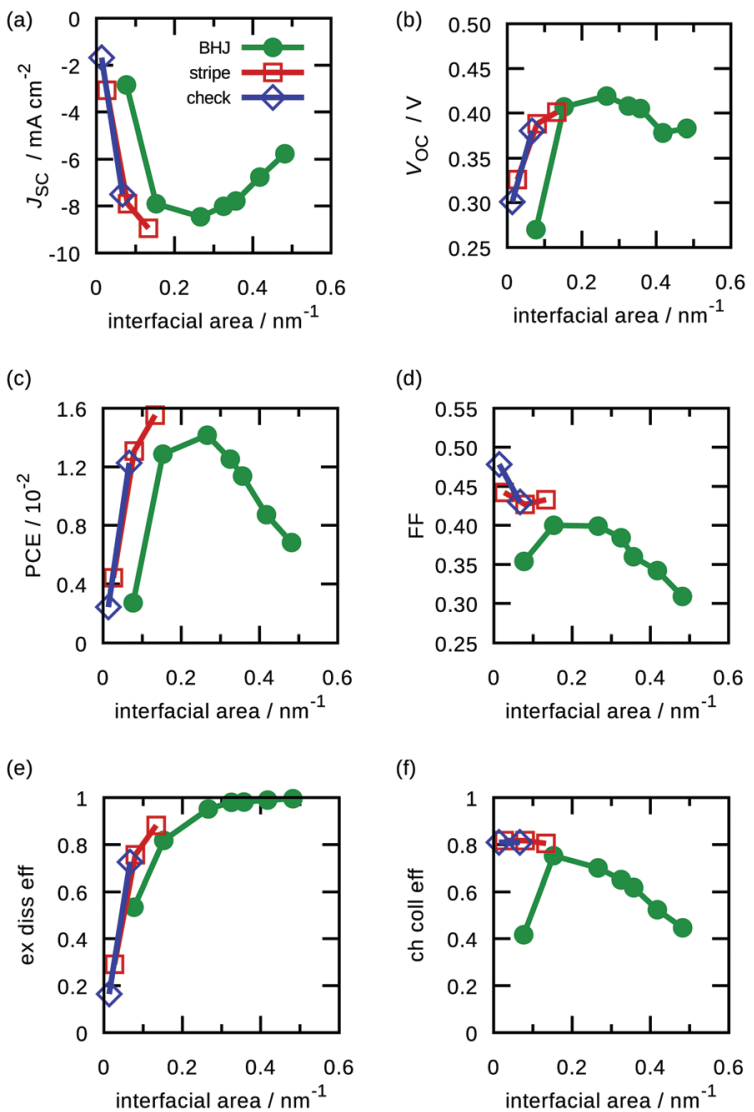

(f)
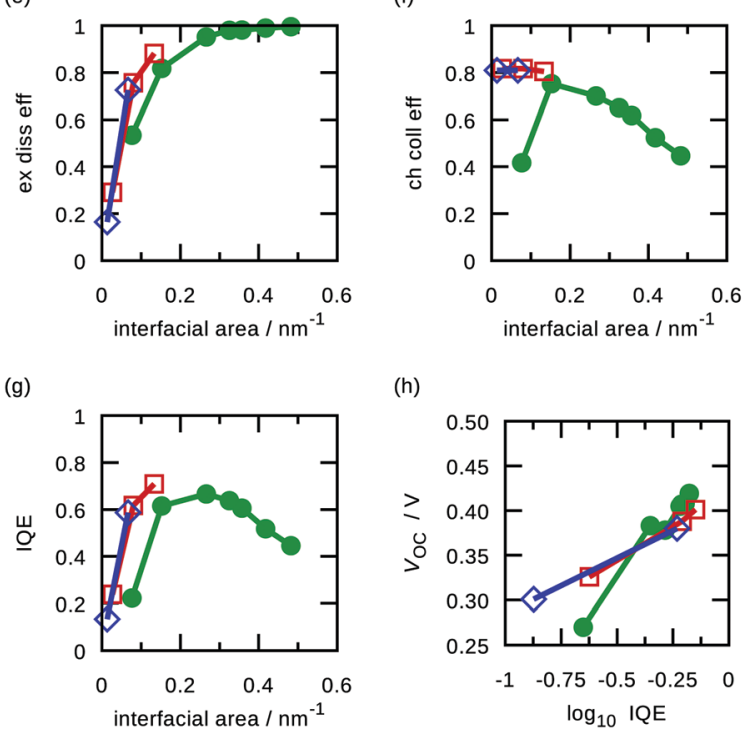

(h)

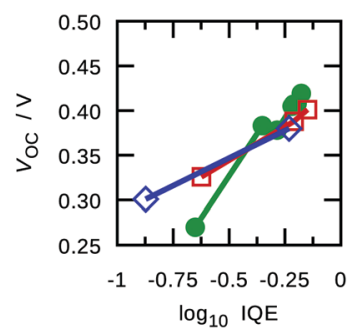

Fig. 8 Interfacial area dependence of performance parameters: (a) shortcircuit current density, (b) open-circuit voltage, (c) power conversion efficiency, (d) fill factor; (e) exciton dissociation efficiency, (f) charge collection efficiency, and (g) IQE. (h) Correlation between $V_{O C}$ and IQE. 
exhibits a maximum value because of the trade-off. These tendencies are consistent with previous studies. ${ }^{27,37,39,40,44}$

In addition, we theoretically demonstrated that the opencircuit voltage $V_{\mathrm{OC}}$ and the logarithm of the IQE have a linear correlation (Fig. 8(h)), which has been reported by an experimental work. ${ }^{18}$ The extrapolated $V_{\text {OC }}$ to $100 \%$ IQE is a little less than $0.5 \mathrm{~V}$, which equals the difference of electrode work functions. Therefore, we revealed that improving the IQE enhances not only $J_{\text {SC }}$ but also $V_{\text {OC }}$.

The dependencies of performance parameters of $\mathrm{BHJ}$ morphologies on $\beta \Delta \varepsilon$, corresponding to finite temperature, are shown in Fig. 9. The morphologies generated under higher $\beta \Delta \varepsilon$ tend to give higher $J_{\mathrm{SC}}$ and $\mathrm{FF}$ with lower $V_{\mathrm{OC}}$. It is shown that $\beta \Delta \varepsilon$ had an optimal value for the PCE-the morphology with $\beta \Delta \varepsilon$ of -2 showed the highest PCE of $1.4 \%$. Remarkably, we revealed that morphology only, without parameter change, determines open-circuit voltage. These effects of thermal annealing are consistent with some experimental results. ${ }^{56,57}$

3.2.2 Transient absorption spectroscopy. The time dependence of the number and peak normalized number of generated charge carriers with 1000 initial excitons is shown in Fig. 10(a) and (b) corresponding to the dynamics of charge generation and recombination, respectively. The simulated number of excitons is shown in Fig. S5 (ESI $\dagger$ ). The standard error of the number of excitons and charge carriers was 10 at the maximum. The tendency was independent of the number of created excitons at the start (see Fig. S6 for the results of 100 initial excitons, ESI $\dagger$ ). The ratio of dissociated to generated excitons varied in a range from $40 \%$ to $90 \%$, consistent with the exciton dissociation efficiencies shown in Fig. 8(e). The time when the number of charge carriers takes the maximum also depended on morphologies: those with lower $\beta \Delta \varepsilon$ tended to show a sudden increase. The morphologies generated with higher temperature tended to
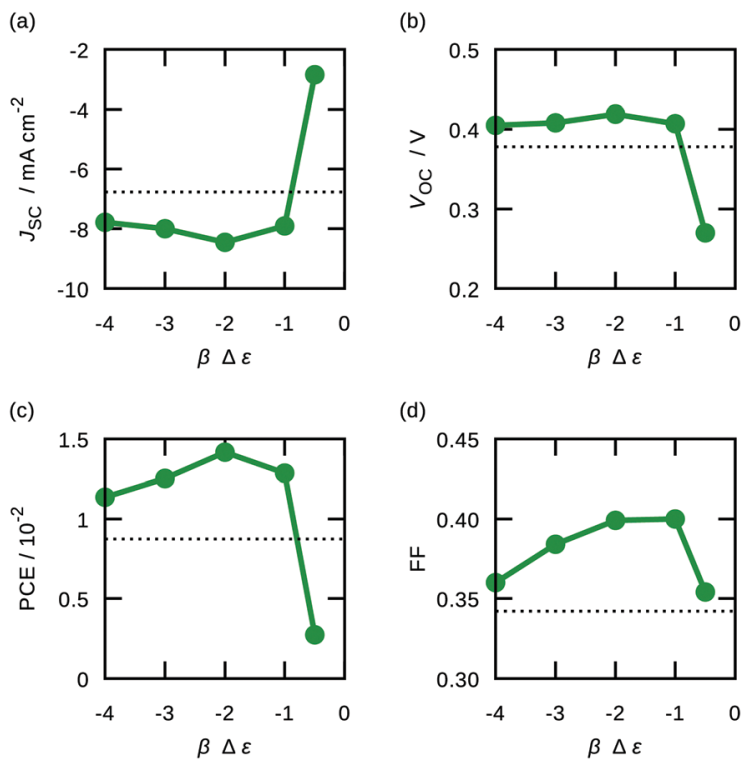

Fig. 9 The dependence of performance parameters of $\mathrm{BHJ}$ morphologies on $\beta \Delta \varepsilon$ : (a) short-circuit current density, $J_{\mathrm{SC}}$ (b) open-circuit voltage, $V_{\mathrm{OC}}$, (c) PCE, and (d) FF. The dashed lines represent those of the initial morphology.
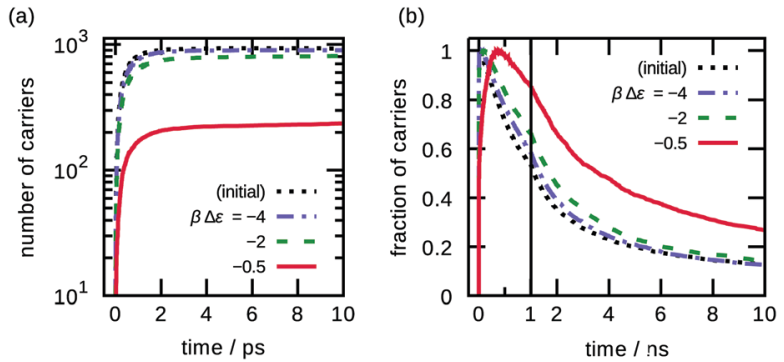

Fig. 10 Simulated charge (a) generation and (b) recombination dynamics of initial structure and morphologies with various $\beta \Delta \varepsilon$.

have larger interfacial areas, and created more charge carriers, whereas they had more bimolecular recombinations, which is due to their large interfacial area. These tendencies are the same as the experimental observations. ${ }^{14}$

The space-time dynamics of charge generation-diffusion length and lifetime of the dissociated excitons-is shown in Fig. 11; see Fig. S7 (ESI $\dagger$ ) for the results of other BHJ morphologies. Note that the lifetime of excitons equals the simulation time. The simulation revealed prompt and delayed dynamics of the charge generation: the majority of the charges (40-95\%, varying for morphologies) were from excitons that were generated on interfaces and promptly (within a few picoseconds) dissociated, corresponding to the sudden increase as shown in Fig. 10; the others were from excitons that migrated to interfaces and dissociated later (up to several hundred nanoseconds), corresponding to the slow increase. The tendency depends on the morphologies because in the case of morphologies of lower temperature, those have higher
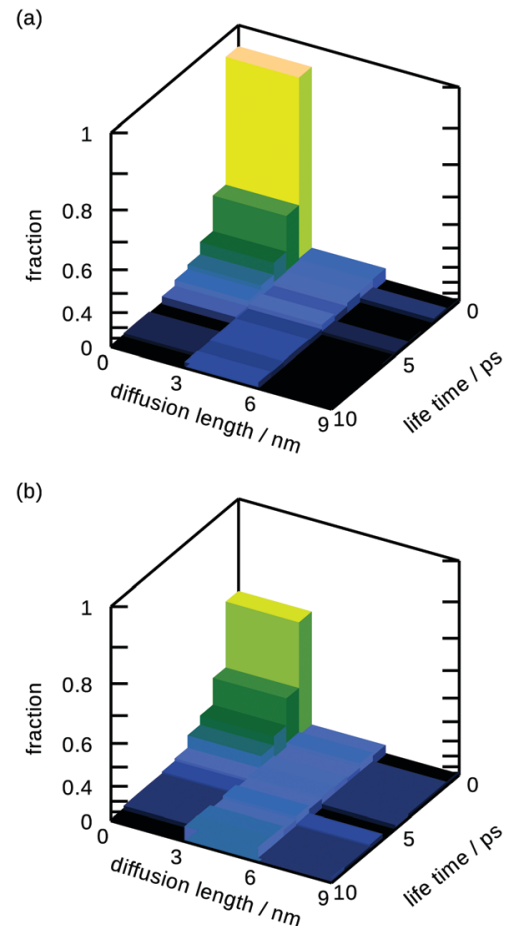

Fig. 11 Fraction of the diffusion length and lifetime of dissociated excitons with a morphology of $\beta \Delta \varepsilon=-1$. 
interfacial area and smaller domains, excitons are more likely generated on a site on an interface and otherwise could reach interfaces easily, resulting in prompt increase of carriers; morphologies of higher temperature show fewer prompt and more delayed exciton dissociation, therefore the number of charges takes the maximum value at a few 100 ps.

\section{Concluding remarks}

We revealed a relationship between the annealing temperature, which is one of the controllable parameters in the manufacturing process, and the performance of OPVs via morphologies of donor and acceptor domains in the active layers. To this end, we proposed a model that combines reptation and DMC. The reptation and DMC were performed to investigate the physical mechanism of formation of the morphologies and the electrochemical profile of photo- and dark currents, and transient absorption spectroscopy, respectively. Then, the reptation with a 1 nm-lattice showed temperature dependencies of morphology. The interfacial area exhibited a minimum value related to the annealing temperature because of the trade-off between thermal diffusion and attractive interaction. The DMC simulations, in which the generated $\mathrm{BHJ}$ morphologies were coarsened to a 3 nm-lattice, revealed morphology dependencies of the photoand dark currents, and OPV performance: short-circuit current density $J_{\mathrm{SC}}$, open-circuit voltage $V_{\mathrm{OC}}$, and PCE exhibit maximum values related to the interfacial area because of the trade-off between exciton dissociation and charge collection. This work theoretically demonstrated the linear correlation between opencircuit voltage $V_{\mathrm{OC}}$ and the logarithm of IQE, which agrees with experiment. Besides, we performed transient absorption spectroscopy of various $\mathrm{BHJ}$ morphologies, which revealed two different dynamics of charge generation-prompt (a few picoseconds) generation from excitons that were generated on interfaces and delayed (order of sub-nanoseconds) generation from excitons which migrated to interfaces; the former is dominant but its ratio depends on the domain size of morphologies.

Coarsening of lattices in the DMC simulations implicitly assumes that electron-hole separation immediately reaches $3 \mathrm{~nm}$, which likely leads to overestimation of the charge separation efficiency and thus shall be verified in future. It is, actually, numerically observed that charge recombination is suppressed in the case of the coarsened morphologies. A mechanism, for example the charge hopping rate increases locally on an interface due to factors like a hot process, ${ }^{58}$ is necessary to compensate it. The assumption and mechanism require further study, which is beyond the scope of this work.

\section{Acknowledgements}

This work was supported by JSPS KAKENHI Grant No. 24750012, CREST JST. We are grateful to Dr A. Ikeda for valuable discussions about polymer dynamics. We appreciate suggestive and stimulating discussions with Buyoung Jung's on DMC algorithm. Theoretical calculations in this study were performed using facilities at the
Supercomputer Center in Institute for Solid State Physics (ISSP) of the University of Tokyo and at Research Center for Computational Science in the Institute for Molecular Science (IMS) in Okazaki, Japan.

\section{References}

1 R. Søndergaard, M. Hösel, D. Angmo, T. T. Larsen-Olsen and F. C. Krebs, Mater. Today, 2012, 15, 36-49.

2 F. C. Krebs, J. Fyenbo, D. M. Tanenbaum, S. A. Gevorgyan, R. Andriessen, B. van Remoortere, Y. Galagan and M. Jorgensen, Energy Environ. Sci., 2011, 4, 4116-4123.

3 N. Espinosa, R. Garcia-Valverde and F. C. Krebs, Energy Environ. Sci., 2011, 4, 1547-1557.

4 M. A. Green, K. Emery, Y. Hishikawa, W. Warta and E. D. Dunlop, Prog. Photovoltaics, 2015, 23, 805-812.

5 F. Liu, Y. Gu, J. W. Jung, W. H. Jo and T. P. Russell, J. Polym. Sci., Part B: Polym. Phys., 2012, 50, 1018-1044.

6 J. A. Bjorgaard and M. E. Kose, RSC Adv., 2015, 5, 8432-8445.

7 G. Yu, J. Gao, J. C. Hummelen, F. Wudl and A. J. Heeger, Science, 1995, 270, 1789-1791.

8 A. J. Pearson, T. Wang, R. A. L. Jones, D. G. Lidzey, P. A. Staniec, P. E. Hopkinson and A. M. Donald, Macromolecules, 2012, 45, 1499-1508.

9 S. S. van Bavel, M. Bärenklau, G. de With, H. Hoppe and J. Loos, Adv. Funct. Mater., 2010, 20, 1458-1463.

10 N. D. Treat, C. G. Shuttle, M. F. Toney, C. J. Hawker and M. L. Chabinyc, J. Mater. Chem., 2011, 21, 15224-15231.

11 H.-C. Liao, C.-S. Tsao, Y.-C. Huang, M.-H. Jao, K.-Y. Tien, C.-M. Chuang, C.-Y. Chen, C.-J. Su, U.-S. Jeng, Y.-F. Chen and W.-F. Su, RSC Adv., 2014, 4, 6246-6253.

12 A. H. Rice, R. Giridharagopal, S. X. Zheng, F. S. Ohuchi, D. S. Ginger and C. K. Luscombe, ACS Nano, 2011, 5, 3132-3140.

13 J. J. van Franeker, D. Westhoff, M. Turbiez, M. M. Wienk, V. Schmidt and R. A. J. Janssen, Adv. Funct. Mater., 2015, 25, 855-863.

14 D. Mori, H. Benten, H. Ohkita and S. Ito, Adv. Energy Mater., 2015, 5, 1500304.

15 T. Shimazaki and T. Nakajima, Phys. Chem. Chem. Phys., 2015, 17, 12538-12544.

16 M. Fujii and K. Yamashita, Chem. Phys. Lett., 2011, 514, 146-150.

17 K. Nishimra, M. Fujii, R. Jono and K. Yamashita, J. Phys. Chem. C, 2015, 119, 26258-26265.

18 M. Fujii, W. Shin, T. Yasuda and K. Yamashita, Phys. Chem. Chem. Phys., 2016, 18, 9514-9523.

19 S. Koda, M. Fujii, S. Hatamiya and K. Yamashita, Theor. Chem. Acc., 2016, 135, 1-10.

20 T. Liu and A. Troisi, J. Phys. Chem. C, 2011, 115, 2406-2415.

21 F. Frigerio, M. Casalegno, C. Carbonera, T. Nicolini, S. V. Meille and G. Raos, J. Mater. Chem., 2012, 22, 5434-5443.

22 S. Obata and Y. Shimoi, Phys. Chem. Chem. Phys., 2013, 15, 9265-9270.

23 B. P. Lyons, N. Clarke and C. Groves, J. Phys. Chem. C, 2011, 115, 22572-22577. 
24 O. Wodo and B. Ganapathysubramanian, Comput. Mater. Sci., 2012, 55, 113-126.

25 A. Pershin, S. Donets and S. A. Baeurle, J. Chem. Phys., 2012, 136, 194102.

26 J. Xue, T. Hou and Y. Li, Appl. Phys. Lett., 2012, 100, 053307.

27 B. P. Lyons, N. Clarke and C. Groves, Energy Environ. Sci., 2012, 5, 7657-7663.

28 M. L. Jones, R. Dyer, N. Clarke and C. Groves, Phys. Chem. Chem. Phys., 2014, 16, 20310-20320.

29 P. G. de Gennes, J. Chem. Phys., 1971, 55, 572-579.

30 M. Doi and S. F. Edwards, J. Chem. Soc., Faraday Trans. 2, 1978, 74, 1789-1801.

31 J. M. Frost, F. Cheynis, S. M. Tuladhar and J. Nelson, Nano Lett., 2006, 6, 1674-1681.

32 K. X. Steirer, P. F. Ndione, N. E. Widjonarko, M. T. Lloyd, J. Meyer, E. L. Ratcliff, A. Kahn, N. R. Armstrong, C. J. Curtis, D. S. Ginley, J. J. Berry and D. C. Olson, Adv. Energy Mater., 2011, 1, 813-820.

33 K. Ishaque, Z. Salam, S. Mekhilef and A. Shamsudin, Appl. Energy, 2012, 99, 297-308.

34 L. J. A. Koster, E. C. P. Smits, V. D. Mihailetchi and P. W. M. Blom, Phys. Rev. B: Condens. Matter Mater. Phys., 2005, 72, 085205.

35 D. Scharfetter and H. Gummel, IEEE Trans. Electron Devices, 1969, 16, 64-77.

36 H. Gummel, IEEE Trans. Electron Devices, 1964, 11, 455-465.

37 P. K. Watkins, A. B. Walker and G. L. B. Verschoor, Nano Lett., 2005, 5, 1814-1818.

38 A. B. Walker, Proc. IEEE, 2009, 97, 1587-1596.

39 L. Meng, Y. Shang, Q. Li, Y. Li, X. Zhan, Z. Shuai, R. G. E. Kimber and A. B. Walker, J. Phys. Chem. B, 2010, 114, 36-41.

40 R. G. E. Kimber, A. B. Walker, G. E. Schroder-Turk and D. J. Cleaver, Phys. Chem. Chem. Phys., 2010, 12, 844-851.

41 K. Feron, C. J. Fell, L. J. Rozanski, B. B. Gong, N. Nicolaidis, W. J. Belcher, X. Zhou, E. Sesa, B. V. King and P. C. Dastoor, Appl. Phys. Lett., 2012, 101, 193306.
42 A. G. Gagorik, J. W. Mohin, T. Kowalewski and G. R. Hutchison, J. Phys. Chem. Lett., 2013, 4, 36-42.

43 C. Groves, Energy Environ. Sci., 2013, 6, 3202-3217.

44 B. Jung, K. Kim, J. Kim, S. Kim, E. Kim and W. Kim, Sol. Energy Mater. Sol. Cells, 2014, 120(Part B), 675-684.

45 C. Groves, R. A. Marsh and N. C. Greenham, J. Chem. Phys., 2008, 129, 114903.

46 M. L. Jones, B. Chakrabarti and C. Groves, J. Phys. Chem. C, 2014, 118, 85-91.

47 D. T. Gillespie, J. Comput. Phys., 1976, 22, 403-434.

48 J. Guo, H. Ohkita, H. Benten and S. Ito, J. Am. Chem. Soc., 2009, 131, 16869-16880.

49 W. C. Tsoi, S. J. Spencer, L. Yang, A. M. Ballantyne, P. G. Nicholson, A. Turnbull, A. G. Shard, C. E. Murphy, D. D. C. Bradley, J. Nelson and J.-S. Kim, Macromolecules, 2011, 44, 2944-2952.

50 H. Sirringhaus, P. J. Brown, R. H. Friend, M. M. Nielsen, K. Bechgaard, B. M. W. Langeveld-Voss, A. J. H. Spiering, R. A. J. Janssen, E. W. Meijer, P. Herwig and D. M. de Leeuw, Nature, 1999, 401, 685-688.

51 M. Chikamatsu, S. Nagamatsu, Y. Yoshida, K. Saito, K. Yase and K. Kikuchi, Appl. Phys. Lett., 2005, 87, 203504.

52 T. Förster, Ann. Phys., 1948, 437, 55-75.

53 A. Miller and E. Abrahams, Phys. Rev., 1960, 120, 745-755.

54 R. A. Marsh, C. Groves and N. C. Greenham, J. Appl. Phys., 2007, 101, 083509.

55 M.-C. Shih, B.-C. Huang, C.-C. Lin, S.-S. Li, H.-A. Chen, Y.-P. Chiu and C.-W. Chen, Nano Lett., 2013, 13, 2387-2392.

56 G. Kalita, M. Masahiro, W. Koichi and M. Umeno, SolidState Electron., 2010, 54, 447-451.

57 W. C. Tsoi, D. T. James, J. S. Kim, P. G. Nicholson, C. E. Murphy, D. D. C. Bradley, J. Nelson and J.-S. Kim, J. Am. Chem. Soc., 2011, 133, 9834-9843.

58 S. M. Falke, C. A. Rozzi, D. Brida, M. Maiuri, M. Amato, E. Sommer, A. De Sio, A. Rubio, G. Cerullo, E. Molinari and C. Lienau, Science, 2014, 344, 1001-1005. 